\title{
A function on the homology of 3-manifolds
}

\author{
VLADIMIR TURAEV
}

\begin{abstract}
In analogy with the Thurston norm, we define for an orientable 3-manifold $M$ a numerical function on $\mathrm{H}_{2}(M ; \mathbb{Q} / \mathbb{Z})$. This function measures the minimal complexity of folded surfaces representing a given homology class. A similar function is defined on the torsion subgroup of $H_{1}(M ; \mathbb{Z})$. These functions are estimated from below in terms of abelian torsions of $M$.
\end{abstract}

57M27; 57Q10

\section{Introduction}

One of the most beautiful invariants of a 3-dimensional manifold $M$ is the Thurston semi-norm on $\mathrm{H}_{2}(M ; \mathbb{Q})$ [9]. The geometric idea leading to this semi-norm is to consider the minimal genus of a surface in $M$ realizing any given 2-homology class of $M$. Thurston's definition of the semi-norm uses a suitably normalized Euler characteristic of the surface rather than the genus. The Thurston semi-norm is uninteresting for a rational homology sphere $M$, since then $H_{2}(M ; \mathbb{Q})=0$. However, a rational homology sphere may have nontrivial 2-homology with coefficients in $\mathbb{Q} / \mathbb{Z}$. Homology classes in $H_{2}(M ; \mathbb{Q} / \mathbb{Z})$ can be realized by folded surfaces, locally looking like unions of several half-planes in $\mathbb{R}^{3}$ with common boundary line. It is natural to consider "smallest" folded surfaces in a given homology class.

We use this train of ideas to define for an arbitrary orientable 3-manifold $M$ (not necessarily a rational homology sphere) a function

$$
\theta=\theta_{M}: H_{2}(M ; \mathbb{Q} / \mathbb{Z}) \rightarrow \mathbb{R}_{+}=\{r \in \mathbb{R} \mid r \geq 0\} .
$$

This function measures the "minimal" normalized Euler characteristic of a folded surface representing a given class in $H_{2}(M ; \mathbb{Q} / \mathbb{Z})$.

Using the boundary homomorphism

$$
d: H_{2}(M ; \mathbb{Q} / \mathbb{Z}) \rightarrow H_{1}(M)=H_{1}(M ; \mathbb{Z}),
$$

whose image is equal to Tors $H_{1}(M)$, we derive from $\theta$ a function

$$
\Theta=\Theta_{M}: \text { Tors } H_{1}(M) \rightarrow \mathbb{R}_{+}
$$


by $\Theta(u)=\inf _{x \in d^{-1}(u)} \theta(x)$ for any $u \in$ Tors $H_{1}(M)$. One can view $\Theta(u)$ as a "normalized minimal genus" of oriented knots in $M$ representing $u$. If $M$ is a rational homology sphere, then $d$ is an isomorphism and $\Theta=\theta \circ d^{-1}$.

We give an estimate of the function $\theta$ from above in terms of the Thurston semi-norm on knot complements in $M$. This estimate implies that $\theta$ is bounded from above and is upper semi-continuous with respect to a natural topology on $H_{2}(M ; \mathbb{Q} / \mathbb{Z}$ ). (I do not know whether $\theta$ is continuous.) The functions $\theta$ and $\Theta$ are also estimated from below using abelian torsions of $M$. These estimates are parallel to the McMullen estimate [6] of the Thurston semi-norm in terms of the Alexander polynomial.

A simple example of nonzero functions $\theta$ and $\Theta$ is provided by the lens space $M=$ $L(5,1)$. We identify $H_{1}(M)=\mathbb{Z} / 5 \mathbb{Z}$ so that the core circles of the two solid tori forming $M$ represents $\pm 1 \in \mathbb{Z} / 5 \mathbb{Z}$. It is shown in Section 2.3, Section 2.4, and Section 6.1 that $\Theta_{M}( \pm 1)=\Theta_{M}(0)=0$ and $\Theta_{M}(2)=\Theta_{M}(-2) \geq 1 / 5$. In this example, the function $\Theta$ takes nonzero values only on $\pm 2 \in \mathbb{Z} / 5 \mathbb{Z}$. This shows that, in contrast to the Thurston semi-norm, the function $\Theta$ may not satisfy the triangle inequality and may be nonhomogeneous, that is in general $\Theta(k x) \neq k \Theta(x)$ for $k \in \mathbb{Z}$ and $x \in H_{1}(M)$. The same remarks apply to $\theta$ since in this example $H_{2}(M ; \mathbb{Q} / \mathbb{Z})=H_{1}(M)$ and $\theta=\Theta \circ d$.

The Thurston semi-norm of a 3-manifold $M$ is fully determined by the Heegaard-Floer homology of $M$ (see Ozsváth and Szabó [8]), and by the Seiberg-Witten monopole homology of $M$ (see Kronheimer and Mrowka [4]). It would be interesting to obtain similar computations of the functions $\theta$ and $\Theta$.

The organization of the paper is as follows. We introduce the functions $\theta$ and $\Theta$ in Section 2 and estimate them from above in Section 3. In Section 4 these functions are estimated from below in the case where the first Betti number of the 3-manifold is nonzero. A similar estimate for rational homology spheres is given in Section 5. In Section 6 we describe a few examples. In Section 7 we make several miscellaneous remarks.

Throughout the paper, the unspecified group of coefficients in homology is $\mathbb{Z}$.

\section{Folded surfaces and the functions $\theta, \Theta$}

\subsection{Folded surfaces}

By a folded surface (without boundary), we mean a compact 2-dimensional polyhedron such that each point has a neighborhood homeomorphic to a union of several half-planes 
in $\mathbb{R}^{3}$ with common boundary line. Such a neighborhood is homeomorphic to $\mathbb{R} \times \Gamma_{n}$ where $n$ is a positive integer and $\Gamma_{n}$ is a union of $n$ closed intervals with one common endpoint and no other common points.

The interior $\operatorname{Int}(X)$ of a folded surface $X$ consists of the points of $X$ which have neighborhoods homeomorphic to $\mathbb{R}^{2}$. Clearly, $\operatorname{Int}(X)$ is a 2-dimensional manifold. The singular set $\operatorname{sing}(X)=X-\operatorname{Int}(X)$ of $X$ consists of a finite number of disjoint circles. A neighborhood of a component of $\operatorname{sing}(X)$ in $X$ fibers over this component with fiber $\Gamma_{n}$ for some $n \neq 2$.

Cutting out $X$ along $\operatorname{sing}(X)$ we obtain a compact 2-manifold (with boundary) $X_{\text {cut }}$. Each component of $\operatorname{Int}(X)$ is the interior of a component of $X_{\text {cut }}$. Set

$$
\chi_{-}(X)=\sum_{Y} \chi_{-}(Y)
$$

where $Y$ runs over all components of $X_{\text {cut }}$ and

$$
\chi_{-}(Y)=\max (-\chi(Y), 0) .
$$

The number $\chi_{-}(X) \geq 0$ measures the complexity of $X$. It is equal to zero if and only if all components of $X_{\text {cut }}$ belong to the following list: spheres, tori, projective planes, annuli, Möbius bands, disks.

By an orientation of a folded surface $X$, we mean an orientation of the 2-manifold $\operatorname{Int}(X)$. An orientation of $X$ allows us to view $X$ as a singular 2-chain with integer coefficients. This $2-$ chain is denoted by the same letter $X$. Its boundary expands as $\sum_{K} i(K)\langle K\rangle$ where $K$ runs over connected components of $\operatorname{sing}(X)$, the symbol $\langle K\rangle$ denotes a 1 -cycle on $K$ representing a generator of $H_{1}(K) \cong \mathbb{Z}$ and $i(K) \in \mathbb{Z}$. Multiplying, if necessary, both $\langle K\rangle$ and $i(K)$ by -1 , we can assume that $i(K) \geq 0$. In this way the integer $i(K)$ is uniquely determined by $K$. It is called the index of $K$ in $X$. For $K$ with $i(K) \neq 0$, the 1 -cycle $\langle K\rangle$ determines an orientation of $K$. We say that this orientation is induced by the one on $X$.

We call a folded surface $X$ simple if it is oriented, the set $\operatorname{sing}(X)$ is homeomorphic to a circle, and its index in $X$ is nonzero. This index is denoted $i_{X}$. Note that $X$ is not required to be connected; however, all components of $X$ but one are closed oriented 2-manifolds.

\subsection{Representation of 2-homology by folded surfaces}

Let $M$ be an orientable 3-manifold. By a folded surface in $M$, we mean a folded surface embedded in $M$. Given a simple folded surface $X$ in $M$, the 2-chain $\left(i_{X}\right)^{-1} X$ 
with rational coefficients is a $2-$ cycle modulo $\mathbb{Z}$. This cycle represents a homology class in $H_{2}(M ; \mathbb{Q} / \mathbb{Z})$ denoted $[X]$.

The short exact sequence of groups of coefficients $0 \rightarrow \mathbb{Z} \rightarrow \mathbb{Q} \rightarrow \mathbb{Q} / \mathbb{Z} \rightarrow 0$ induces an exact homology sequence:

$$
\cdots \rightarrow H_{2}(M ; \mathbb{Q}) \rightarrow H_{2}(M ; \mathbb{Q} / \mathbb{Z}) \rightarrow H_{1}(M) \rightarrow H_{1}(M ; \mathbb{Q}) \rightarrow \cdots
$$

The homomorphism $H_{2}(M ; \mathbb{Q} / \mathbb{Z}) \rightarrow H_{1}(M)$ in this sequence will be denoted $d_{M}$ and called the boundary homomorphism. The exactness of (1) implies that the image of $d_{M}$ is equal to the group Tors $H_{1}(M)$ consisting of all elements of $H_{1}(M)$ of finite order.

For a simple folded surface $X$ in $M$, the homomorphism $d_{M}$ sends $[X]$ into the homology class in $H_{1}(M)$ represented by the circle $\operatorname{sing}(X)$ with orientation induced by the one on $X$.

For example, if $X \subset M$ is a compact oriented 2-manifold with connected nonvoid boundary, then $X$ is a simple folded surface with $\operatorname{sing}(X)=\partial X, i_{X}=1$, and $[X]=0$. Another example: consider an unknotted circle $K$ lying in a 3-ball in $M$ and pick $n \neq 2$ closed 2 -disks bounded by $K$ in this ball and having no other common points. We orient these disks so that the induced orientations on $K$ are the same. The union of these disks, $X=X(n)$, is a simple folded surface with $\operatorname{sing}(X)=K, i_{X}=n$, and $[X]=0$.

Lemma 2.1 Any homology class $x \in H_{2}(M ; \mathbb{Q} / \mathbb{Z})$ can be represented by a simple folded surface.

Proof Set $d=d_{M}: H_{2}(M ; \mathbb{Q} / \mathbb{Z}) \rightarrow H_{1}(M)$. We can represent $d(x) \in$ Tors $H_{1}(M)$ by an oriented embedded circle $K \subset \operatorname{Int}(M)=M-\partial M$. Pick an integer $n \geq 1$ such that $n d(x)=0$. The standard arguments, using the Poincaré duality and transversality, show that there is a simple folded surface $X$ in $M$ such that $\operatorname{sing}(X)=K$ and $i_{X}=n$.

Since both $X$ and $M$ are orientable, the 1-dimensional normal bundle of $\operatorname{Int}(X)$ in $M$ is trivial. Keeping $\operatorname{sing}(X)$ and pushing $X-\operatorname{sing}(X)$ in a normal direction, we obtain a "parallel" copy $X_{1}$ of $X$ such that $X \cap X_{1}=\operatorname{sing}\left(X_{1}\right)=\operatorname{sing}(X)=K$. The orientation of $X$ induces an orientation of $X_{1}$ in the obvious way. Repeating this process $k \geq 1$ times, we can obtain $k$ parallel copies $X_{1}, X_{2}, \ldots, X_{k}$ of $X$ meeting each other exactly at $K$. Then $X^{(k)}=X_{1} \cup X_{2} \cup \ldots \cup X_{k}$ is a simple folded surface such that $\operatorname{sing}\left(X^{(k)}\right)=K$ and $i_{X^{(k)}}=n k$. It follows from the construction that $\left[X^{(k)}\right]=[X] \in H_{2}(M ; \mathbb{Q} / \mathbb{Z})$ for all $k \geq 1$. 
The equalities $d(x)=[K]=d([X])$ imply that $x-[X] \in \operatorname{Ker} d=\operatorname{Im} j$, where $j$ is the homomorphism $H_{2}(M ; \mathbb{Q}) \rightarrow H_{2}(M ; \mathbb{Q} / \mathbb{Z})$ induced by the projection $\mathbb{Q} \rightarrow \mathbb{Q} / \mathbb{Z}$. Pick $y \in j^{-1}(x-[X]) \subset H_{2}(M ; \mathbb{Q})$. There is an integer $k \geq 1$ such that $k y$ lies in the image of the coefficient homomorphism $H_{2}(M ; \mathbb{Z}) \rightarrow H_{2}(M ; \mathbb{Q})$. A fortiori, the homology class $n k y$ lies in this image. We represent $n k y$ by a closed oriented (possibly nonconnected) surface $\Sigma \subset M$. Since $d(x) \in$ Tors $H_{1}(M)$, the intersection number $\Sigma \cdot K=\Sigma \cdot d(x)$ is 0 . Applying if necessary surgeries of index 1 to $\Sigma$, we can assume that $\Sigma \cap K=\varnothing$. Then $y$ is represented by the 2 -cycle $(n k)^{-1} \Sigma$ in $M-K$ and $x=[X]+j(y)=\left[X^{(k)}\right]+j(y)$ is represented by the 2 -cycle $(n k)^{-1}\left(X^{(k)}+\Sigma\right)$ $\bmod \mathbb{Z}$. Applying to $X^{(k)}$ and $\Sigma$ the usual cut and paste technique, we can transform their union into a simple folded surface $Z$ such that $\operatorname{sing}(Z)=\operatorname{sing}\left(X^{(k)}\right)=K$ and $i_{Z}=n k$. Clearly, $[Z]=x$.

\subsection{Functions $\theta$ and $\Theta$}

For an orientable 3-dimensional manifold $M$, we define a function $\theta=\theta_{M}$ from $H_{2}(M ; \mathbb{Q} / \mathbb{Z})$ to $\mathbb{R}_{+}$by

$$
\theta(x)=\inf _{X} \frac{\chi_{-}(X)}{i_{X}},
$$

where $x \in H_{2}(M ; \mathbb{Q} / \mathbb{Z})$ and $X$ runs over all simple folded surfaces in $M$ representing $x$. In particular, the class $x=0$ can be represented by the simple folded surface $X=X(n) \subset M$ with $n \neq 2$, constructed before Lemma 2.1. The equality $\chi_{-}(X)=0$ implies that $\theta(0)=0$.

For a simple folded surface $X$, denote by $-X$ the same simple folded surface with opposite orientation in its interior. The obvious equalities

$$
[-X]=-[X], \quad \chi_{-}(-X)=\chi_{-}(X), \quad i_{-X}=i_{X}
$$

imply that $\theta(-x)=\theta(x)$ for all $x \in H_{2}(M ; \mathbb{Q} / \mathbb{Z})$.

We define a function $\Theta=\Theta_{M}$ : Tors $H_{1}(M) \rightarrow \mathbb{R}_{+}$by

$$
\Theta(u)=\inf _{x \in d^{-1}(u)} \theta(x)=\inf _{X} \frac{\chi_{-}(X)}{i_{X}},
$$

where $u \in$ Tors $H_{1}(M), X$ runs over all simple folded surfaces in $M$ such that the circle $\operatorname{sing}(X)$ represents $u$, and $d: H_{2}(M ; \mathbb{Q} / \mathbb{Z}) \rightarrow H_{1}(M)$ is the boundary homomorphism. In (3), we can restrict ourselves to connected $X$. Indeed, all components of $X$ disjoint from $\operatorname{sing}(X)$ are closed oriented surfaces. They may be removed from $X$ without increasing $\chi_{-}(X)$.

Algebraic ${ }^{3} \mathcal{G}$ Geometric Topology, Volume 7 (2007) 
The properties of $\theta$ imply that $\Theta(0)=0$ and $\Theta(-u)=\theta(u)$ for all $u \in$ Tors $H_{1}(M)$. By the very definition of $\Theta$, for all $x \in H_{2}(M ; \mathbb{Q} / \mathbb{Z})$,

$$
\theta(x) \geq \Theta(d(x))
$$

Using folded surfaces with boundary, we can similarly define relative versions

$$
H_{2}(M, \partial M ; \mathbb{Q} / \mathbb{Z}) \rightarrow \mathbb{R}_{+} \quad \text { and } \quad \text { Tors } H_{1}(M, \partial M ; \mathbb{Z}) \rightarrow \mathbb{R}_{+}
$$

of the functions $\theta$ and $\Theta$. We will not study them in this paper.

\subsection{Constructions and examples}

2.4.1 Let $\Sigma$ be a closed connected 2-manifold embedded in an oriented 3-manifold $M$. Let $K \subset \Sigma$ be a simple closed curve such that $\Sigma-K$ has an orientation which switches to the opposite when one crosses $K$ in $\Sigma$. (Such an orientation exists when $\Sigma$ is orientable and $K$ splits $\Sigma$ into two surfaces or when $\Sigma$ is nonorientable and $K$ represents the Stiefel-Whitney class $w^{1}(\Sigma) \in H^{1}(\Sigma ; \mathbb{Z} / 2 \mathbb{Z})=H_{1}(\Sigma ; \mathbb{Z} / 2 \mathbb{Z})$.) The orientations of $M$ and $\Sigma-K$ induce an orientation of the normal bundle of $\Sigma-K$ in $M$. Keeping $K$ and pushing $\Sigma-K$ in the corresponding normal direction, we obtain a copy $\Sigma^{\prime}$ of $\Sigma$ such that $\Sigma^{\prime}$ transversely meets $\Sigma$ along $K$. The union $X=\Sigma \cup \Sigma^{\prime}$ is a simple folded surface such that $\operatorname{sing}(X)=K$ and $i_{X}=4$. Then $\theta([X]) \leq(1 / 4) \chi_{-}(X)=(1 / 2) \chi_{-}(\Sigma-K)$.

For example, we can apply this construction to the projective plane $\Sigma=\mathbb{R} P^{2}$ in $\mathbb{R} P^{3}$ taking as $K$ a projective circle on $\mathbb{R} P^{2}$. The resulting simple folded surface $X$ represents the only nonzero element $x$ of $H_{2}\left(\mathbb{R} P^{3} ; \mathbb{Q} / \mathbb{Z}\right)=\mathbb{Z} / 2 \mathbb{Z}$ because $\operatorname{sing}(X)$ represents the nonzero element of $H_{1}\left(\mathbb{R} P^{3}\right)=\mathbb{Z} / 2 \mathbb{Z}$. The equality $\chi_{-}(\Sigma-K)=0$ implies that $\theta_{\mathbb{R} P^{3}}=0$ and $\Theta_{\mathbb{R} P^{3}}=0$.

2.4.2 Consider the 3-dimensional lens space $M=L(p, q)$, where $p, q$ are coprime integers with $p \geq 2$. The manifold $M$ splits as a union of two solid tori with common boundary. It is easy to exhibit a folded surface $X \subset M$ such that $\operatorname{sing}(X)$ is the core circle of one of the solid tori and $X-\operatorname{sing}(X)$ is a disjoint union of $p$ open 2-disks. This implies that the function $\Theta_{M}$ annihilates the elements of $H_{1}(M)$ represented by the core circles of the solid tori. Under an appropriate isomorphism $H_{1}(M) \cong \mathbb{Z} / p \mathbb{Z}$, these elements correspond to $1(\bmod p)$ and $q(\bmod p)$. This implies that $\Theta_{M}=0$ if $p=2$ or $p=3$ or $p=5, q=2$. For $p=2$, we recover the previous example, since $L(2,1)=\mathbb{R} P^{3}$. 
2.4.3 Let $K$ be an oriented homologically trivial knot in an oriented 3-manifold $N$. Let $M$ be obtained by a $(p, q)$-surgery on $K$ where $p, q$ are coprime integers with $p \geq 2$. Thus, $M$ is obtained by cutting out a tubular neighborhood $U \subset N$ of $K$ and gluing it back along a homeomorphism $\partial U \rightarrow \partial U$ mapping the meridian $\mu \subset \partial U$ of $K$ onto a curve on $\partial U$ homological to $p \mu+q \lambda$, where $\lambda \subset \partial U$ is the longitude of $K$ homologically trivial in $N-K$. The element $u \in H_{1}(M)$ represented by the (oriented) core circle of the solid torus $U \subset M$ has finite order. This follows from the fact that the $p$-th power of the core circle is homotopic in $U \subset M$ to $\lambda \subset \partial U$. We claim that $\Theta(u)=0$ if $K$ is a trivial knot in $N$ and $\Theta(u) \leq p^{-1}(2 g-1)$ if $K$ is a nontrivial knot of genus $g \geq 1$. Indeed, the longitude $\lambda$ bounds in $N-\operatorname{Int}(U)$ an embedded compact connected oriented surface of genus $g$. This surface extends in the obvious way to a simple folded surface $X$ in $M$ such that $\operatorname{sing}(X)$ is the core circle of $U \subset M$ and $i_{X}=p$. Clearly, $\chi_{-}(X)=\max (2 g-1,0)$. This implies our claim. (For $p=2$, one should "double" $X$ along $\operatorname{sing}(X)$ as in Section 2.4.1.) As we shall see below, if $K$ is a nontrivial fibred knot and $p \geq 4 g-2$, then $\Theta(u)=p^{-1}(2 g-1)$.

\section{Estimates from above and semi-continuity}

In this section we estimate the function $\theta=\theta_{M}$ from above using the Thurston norm. Throughout this section, $M$ is a connected orientable 3-manifold (possibly, noncompact).

\subsection{Comparison with the Thurston norm}

Recall first the definition of the Thurston semi-norm $\|\cdot\|_{M}$ on $H_{2}(M ; \mathbb{Q})$. The Poincare duality (applied to compact submanifolds of $M$ ) implies that the abelian group $H_{2}(M)=H_{2}(M ; \mathbb{Z})$ has no torsion. We shall view $H_{2}(M)$ as a lattice in the $\mathbb{Q}$-vector space $H_{2}(M ; \mathbb{Q})=\mathbb{Q} \otimes_{\mathbb{Z}} H_{2}(M)$. For any $x \in H_{2}(M ; \mathbb{Q})$, there is an integer $n \geq 1$ such that $n x \in H_{2}(M)$. Then $\|x\|_{M}=n^{-1} \min _{\Sigma} \chi_{-}(\Sigma) \in \mathbb{Q}$, where $\Sigma$ runs over all closed oriented embedded surfaces in $M$ representing $n x$. The number $\|x\|_{M}$ does not depend on the choice of $n$ and is always realized by a certain $\Sigma$. Using surfaces in $M$ with boundary on $\partial M$, one similarly defines the Thurston semi-norm on $H_{2}(M, \partial M ; \mathbb{Q})$.

Lemma 3.1 Let $j$ be the coefficient homomorphism $H_{2}(M ; \mathbb{Q}) \rightarrow H_{2}(M ; \mathbb{Q} / \mathbb{Z})$. Then $\theta(j(x)) \leq\|x\|_{M}$ for any $x \in H_{2}(M ; \mathbb{Q})$.

Proof Let $\Sigma$ be a closed oriented embedded surface in $M$ representing $n x \in H_{2}(M)$ with $n \geq 3$. The surface $\Sigma$ is an oriented folded surface with empty singular set. 
Consider a folded surface $X=X(n)$ inside a 3-ball in $M-\Sigma$, as constructed before Lemma 2.1. The union $Z=X \cup \Sigma$ is a simple folded surface representing $x$ and $i_{Z}=i_{X}=n$. By the definition of $\theta$,

$$
\theta(j(x)) \leq n^{-1} \chi_{-}(Z)=n^{-1} \chi_{-}(\Sigma) .
$$

Therefore $\theta(j(x)) \leq\|x\|_{M}$.

Lemma 3.2 Let $K$ be an oriented knot in $M$. Set $N=M-K$ and let $\iota$ be the inclusion homomorphism $\mathrm{H}_{2}(N ; \mathbb{Q}) \rightarrow H_{2}(M ; \mathbb{Q})$. Let $j$ be the coefficient homomorphism $H_{2}(M ; \mathbb{Q}) \rightarrow H_{2}(M ; \mathbb{Q} / \mathbb{Z})$. Then for any simple folded surface $X$ in $M$ with $\operatorname{sing}(X)=K$ and any $y \in H_{2}(N ; \mathbb{Q})$,

$$
\theta([X]+j \iota(y)) \leq\left(i_{X}\right)^{-1} \chi_{-}(X)+\|y\|_{N} .
$$

Proof Set $n=i_{X}$ and let $k$ be a positive integer such that $k y \in H_{2}(N) \subset H_{2}(N ; \mathbb{Q})$. It is enough to prove that for any closed oriented surface $\Sigma \subset N$ representing $n k y$,

$$
\theta([X]+j \iota(y)) \leq n^{-1} \chi_{-}(X)+(n k)^{-1} \chi_{-}(\Sigma) .
$$

This can be reformulated in terms of the simple folded surface $X^{(k)}$ as

$$
\theta\left(\left[X^{(k)}\right]+j \iota(y)\right) \leq(n k)^{-1}\left(\chi_{-}\left(X^{(k)}\right)+\chi_{-}(\Sigma)\right) .
$$

Therefore it is enough to prove that for any simple folded surface $T$ in $M$ with $\operatorname{sing}(T)=K$ and $i_{T}=n k$,

$$
\theta([T]+j \iota(y)) \leq(n k)^{-1}\left(\chi_{-}(T)+\chi_{-}(\Sigma)\right) .
$$

Suppose first that $T$ is compressible in $N=M-K$ in the sense that there is an embedded closed 2-disk $D \subset N$ such that $T \cap D=\partial D \subset T-K$ and the circle $\partial D$ does not bound a 2-disk in $T-K$. The surgery on $T$ along $D$ yields a simple folded surface $T_{D}$ with $\left[T_{D}\right]=[T]$ and $\chi_{-}\left(T_{D}\right)<\chi_{-}(T)$. Applying this procedure several times, we can reduce (6) to the case where $T$ is incompressible, ie $T$ admits no disks $D$ as above. By the same reasoning, we can assume that $\Sigma$ is incompressible in $N$ (it may be compressible in $M)$. The homology class $[T]+j \iota(y) \in H_{2}(M ; \mathbb{Q} / \mathbb{Z})$ is represented by the $2-$ cycle $(n k)^{-1} T \cup \Sigma(\bmod \mathbb{Z})$. Deforming $\Sigma$ in $N$ so that it meets $T$ transversely and applying to $T \cup \Sigma$ the usual cut and paste technique, we can transform $T \cup \Sigma$ into a simple folded surface $Z$ with $\operatorname{sing}(Z)=\operatorname{sing}(T)=K$ and $i_{Z}=n k$. Clearly, $[Z]=[T]+j \iota(y)$. The folded surface $Z$ may have spherical components (that is components homeomorphic to $S^{2}$ ) created from pieces of $T-K$ and $\Sigma$ by cutting and pasting. One of these pieces will necessarily be a $2-$ disk $D$ such that either $D \subset T-K$ and $D \cap \Sigma=\partial D$ or $D \subset \Sigma$ and $D \cap(T-K)=\partial D$. 
In the first case the incompressibility of $\Sigma$ implies that the circle $\partial D$ bounds a disk on $\Sigma$. The surgery on $\Sigma$ along $D$ yields a surface $\Sigma_{+} \approx \Sigma \amalg S^{2}$ homological to $\Sigma$ in $N$. Then $\chi_{-}\left(\Sigma_{+}\right)=\chi_{-}(\Sigma)$ and the 1-manifold $T \cap \Sigma_{+}$has one component less than $T \cap \Sigma$. Similarly, if $D \subset \Sigma$, then the incompressibility of $T-K$ implies that $\partial D$ bounds a disk on $T-K$. The surgery on $T$ along $D$ yields a simple folded surface $T_{+} \approx T \amalg S^{2}$ such that $\left[T_{+}\right]=[T], \chi_{-}\left(T_{+}\right)=\chi_{-}(T)$, and the 1-manifold $T_{+} \cap \Sigma$ has one component less than $T \cap \Sigma$. Continuing in this way, we can reduce ourselves to the case where $Z$ does not have spherical components except the spherical components of $T$ disjoint from $\Sigma$ and the spherical components of $\Sigma$ disjoint from $T$. A similar argument allows us to assume that the components of $Z-K$ are not disks except the disk components of $T-K$ disjoint from $\Sigma$. Then the additivity of the Euler characteristic under cutting and pasting implies that $\chi_{-}(Z)=\chi_{-}(T)+\chi_{-}(\Sigma)$. Therefore

$$
\theta([T]+j \iota(y)) \leq(n k)^{-1} \chi_{-}(Z)=(n k)^{-1}\left(\chi_{-}(T)+\chi_{-}(\Sigma)\right) .
$$

This proves (6), (5), and (4).

Theorem 3.3 If $M$ is compact, then there is a number $C>0$ (depending on $M$ ) such that $\theta(x) \leq C$ for all $x \in H_{2}(M ; \mathbb{Q} / \mathbb{Z})$.

Proof Set $d=d_{M}: H_{2}(M ; \mathbb{Q} / \mathbb{Z}) \rightarrow H_{1}(M)$. Since the group $\operatorname{Im} d=$ Tors $H_{1}(M)$ is finite, it is enough to prove that for every $u \in$ Tors $H_{1}(M)$, the values of $\theta$ on the elements of the set $d^{-1}(u)$ are bounded from above.

Consider first the case $u=0$. Then $d^{-1}(u)=\operatorname{Im} j$ where $j$ is the coefficient homomorphism $H_{2}(M ; \mathbb{Q}) \rightarrow H_{2}(M ; \mathbb{Q} / \mathbb{Z})$. We need to prove that the values of $\theta \circ j$ are bounded from above. Since $M$ is compact, the group $H_{2}(M)$ is finitely generated. Pick a basis $a_{1}, \ldots, a_{n}$ in $H_{2}(M)$ and let $Q \subset H_{2}(M ; \mathbb{Q})$ be the cube consisting of the vectors $r_{1} a_{1}+\ldots+r_{n} a_{n}$ with rational nonnegative $r_{1}, \ldots, r_{n} \leq 1$. The supremum $s=\sup _{x \in Q}\|x\|_{M}$ is a finite number, because the Thurston semi-norm extends to a continuous semi-norm on $H_{2}(M ; \mathbb{R})$ and the closure of $Q$ in $H_{2}(M ; \mathbb{R})$ is compact. We claim that $\theta(j(x)) \leq s$ for any $x \in H_{2}(M ; \mathbb{Q})$. Indeed, there is $a \in H_{2}(M)$ such that $x+a \in Q$. Then $j(x)=j(x+a)$ and $\theta(j(x))=\theta(j(x+a)) \leq s$.

Consider now the case $u \neq 0$. Pick an oriented knot $K \subset M$ representing $u$ and a simple folded surface $X$ in $M$ with $\operatorname{sing}(X)=K$. Then $d^{-1}(u)=\{[X]+j \iota(y)\} y$ where $\iota$ is the inclusion homomorphism $H_{2}(M-K ; \mathbb{Q}) \rightarrow H_{2}(M ; \mathbb{Q})$ and $y$ runs over $H_{2}(M-K ; \mathbb{Q})$. The rest of the argument goes as in the case $u=0$ using Lemma 3.2. 


\subsection{Semi-continuity}

For compact $M$, the group $H_{2}(M ; \mathbb{Q} / \mathbb{Z})$ has a natural topology as follows. The image of the coefficient homomorphism $j: H_{2}(M ; \mathbb{Q}) \rightarrow H_{2}(M ; \mathbb{Q} / \mathbb{Z})$ can be identified with the quotient $H_{2}(M ; \mathbb{Q}) / H_{2}(M)$. Provide $\operatorname{Im}(j)$ with the quotient topology induced by the standard topology in the finite dimensional $\mathbb{Q}$-vector space $\mathrm{H}_{2}(M ; \mathbb{Q})$. This extends to a topology in $H_{2}(M ; \mathbb{Q} / \mathbb{Z})$ by declaring a set $U \subset H_{2}(M ; \mathbb{Q} / \mathbb{Z})$ open if $(a+U) \cap \operatorname{Im}(j)$ is open in $\operatorname{Im}(j)$ for all $a \in H_{2}(M ; \mathbb{Q} / \mathbb{Z})$. Recall that an $\mathbb{R}$-valued function $f$ on a topological space $A$ is upper semi-continuous if for any point $a \in A$ and any real $\varepsilon>0$, there is a neighborhood $U \subset A$ of $a$ such that $f(U) \subset(-\infty, f(a)+\varepsilon)$.

Lemma 3.4 For compact $M$, the function $\theta=\theta_{M}$ is upper semi-continuous.

Proof Let $a \in H_{2}(M ; \mathbb{Q} / \mathbb{Z})$ and $\varepsilon>0$. Let $X$ be a simple folded surface in $M$ representing $a$ and such that $\left(i_{X}\right)^{-1} \chi_{-}(X) \leq \theta(a)+\varepsilon / 2$. Set $K=\operatorname{sing}(X)$ and $N=M-K$. Let $\iota: H_{2}(N ; \mathbb{Q}) \rightarrow H_{2}(M ; \mathbb{Q})$ be the inclusion homomorphism. Put

$$
V=\left\{y \in H_{2}(N ; \mathbb{Q}) \mid\|y\|_{N}<\varepsilon / 2\right\} .
$$

The set $V$ is open in $H_{2}(N ; \mathbb{Q})$ since the Thurston norm is continuous. The set $\iota(V)$ is open in $H_{2}(M ; \mathbb{Q})$ since $\iota$ is an epimorphism. The set $j \iota(V)$ is open in $\operatorname{Im}(j)$ by definition of the topology in $\operatorname{Im}(j)$. Finally, the set $U=a+j \iota(V)$ is an open neighborhood of $a$ in $H_{2}(M ; \mathbb{Q} / \mathbb{Z})$ by definition of the topology in $H_{2}(M ; \mathbb{Q} / \mathbb{Z})$. By (4), we have $\theta(U) \subset(-\infty, \theta(a)+\varepsilon)$. Hence $\theta$ is upper semi-continuous.

\section{Estimates from below: the case $b_{1} \geq 1$}

In this section we give an estimate from below for the functions $\theta=\theta_{M}$ and $\Theta=\Theta_{M}$ of a 3-manifold $M$ with nonzero first Betti number $b_{1}(M)$. We begin with preliminaries on group rings and abelian torsions of 3-manifolds.

\subsection{Preliminaries}

Let $H$ be a finitely generated abelian group written in multiplicative notation. Any element $a$ of the group ring $\mathbb{Q}[H]$ expands uniquely in the form $a=\sum_{h \in H} a_{h} h$, where $a_{h} \in \mathbb{Q}$ and $a_{h}=0$ for all but finitely many $h$. We say that an element $h \in H$ is $a$-basic if $a_{h} \neq 0$. The (finite) set of $a$-basic elements of $H$ is denoted $B_{a}$. The element $\sum_{h \in \text { Tors } H} h$ of $\mathbb{Q}[H]$ will be denoted $\Sigma_{H}$. Clearly, $B_{\Sigma_{H}}=$ Tors $H$. 
The classical ring of quotients of $\mathbb{Q}[H]$ that is, the (commutative) ring obtained by inverting all nonzero-divisors of $\mathbb{Q}[H]$ is denoted $Q(H)$. It is known that $\mathbb{Q}[H]$ splits as a direct sum of domains. Therefore $Q(H)$ splits as a direct sum of fields and the natural ring homomorphism $\mathbb{Q}[H] \rightarrow Q(H)$ is an embedding. We identify $\mathbb{Q}[H]$ with its image under this embedding. Note that if $H$ is a finite abelian group, then $Q(H)=\mathbb{Q}[H]$.

Let $M$ be a compact connected 3-manifold. From now on, we use multiplicative notation for the group operation in $H=H_{1}(M)$. In particular, the neutral element of $H$ is denoted 1 . The manifold $M$ gives rise to a maximal abelian torsion $\tau(M)$ which is an element of $Q(H)$ defined up to multiplication by -1 and elements of $H$ (see Turaev [11] and Nicolaescu [7]). If $b_{1}(M) \geq 2$, then all representatives of $\tau(M)$ belong to $\mathbb{Z}[H] \subset \mathbb{Q}[H] \subset Q(H)$. We express this by writing $\tau(M) \in \mathbb{Z}[H]$. If $b_{1}(M)=1$ and $\partial M \neq \varnothing$, then $\tau(M) \in \mathbb{Z}[H]+\Sigma_{H} \cdot Q(H)$. This implies that $(h-1) \tau(M) \in \mathbb{Z}[H]$ for all $h \in$ Tors $H$ (indeed $\left.(h-1) \Sigma_{H}=0\right)$.

If $M$ is oriented and $b_{1}(M) \geq 2$, then the Thurston semi-norm $\|\cdot\|_{M}$ on $H_{2}(M, \partial M ; \mathbb{Q})$ can be estimated in terms of $\tau(M)$ as follows (see [11]): for any $s \in H_{2}(M, \partial M ; \mathbb{Q}$ ) and any representative $a \in \mathbb{Z}[H]$ of $\tau(M)$,

$$
\|s\|_{M} \geq \max _{h, h^{\prime} \in B_{a}}\left|h \cdot s-h^{\prime} \cdot s\right|,
$$

where $h \cdot s \in \mathbb{Z}$ is the intersection index of $h$ and $s$. Note that the right hand side of (7) does not depend on the choice of $a$ in $\tau(M)$.

\subsection{An estimate for $\theta_{M}$}

The function $\theta$ will be estimated in terms of spans of subsets of $\mathbb{Q} / \mathbb{Z}$. The span $\operatorname{spn}(A)$ of a finite set $A \subset \mathbb{Q} / \mathbb{Z}$ is a rational number defined as the minimal length of an interval in $\mathbb{Q} / \mathbb{Z}$ containing $A$, that is the minimal rational number $t \geq 0$ such that for some $r \in \mathbb{Q}$, the projection of the set $[r, r+t] \cap \mathbb{Q}$ into $\mathbb{Q} / \mathbb{Z}$ contains $A$. Clearly, $1>\operatorname{spn}(A) \geq 0$ and $\operatorname{spn}(A)=0$ if and only if $A$ is empty or has only one element.

Given an oriented 3-manifold $M$ and a homology class $x \in H_{2}(M ; \mathbb{Q} / \mathbb{Z})$, we set for any $a \in \mathbb{Q}\left[H_{1}(M)\right]$,

$$
\operatorname{spn}_{x}(a)=\operatorname{spn}\left(\{h \cdot x\}_{h \in B_{a}}\right),
$$

where $h \cdot x \in \mathbb{Q} / \mathbb{Z}$ is the intersection index of $h$ and $x$. Clearly, $1>\operatorname{spn}_{x}(a) \geq 0$.

Theorem 4.1 Let $M$ be a compact connected oriented 3-manifold with $b_{1}(M) \geq 1$. Set $H=H_{1}(M)$ and let $\tau \in Q(H)$ be a representative of the torsion $\tau(M)$. Let $x \in H_{2}(M ; \mathbb{Q} / \mathbb{Z})$ and $u=d_{M}(x) \in H$. Then $(u-1) \tau \in \mathbb{Z}[H]$ and

$$
\theta_{M}(x) \geq \operatorname{spn}_{x}((u-1) \tau) .
$$


Proof If $b_{1}(M) \geq 2$, then $\tau \in \mathbb{Z}[H]$ and $(u-1) \tau \in \mathbb{Z}[H]$. The inclusion $u \in$ Tors $H$ and the remarks in Section 4.1 imply that $(u-1) \tau \in \mathbb{Z}[H]$ for $b_{1}(M)=1$ as well.

We prove (8). Let $X$ be a simple folded surface in $M$ representing $x$. The knot $\operatorname{sing}(X) \subset M$ endowed with orientation induced from the one on $X$ represents the class $u \in$ Tors $H$. Let $E$ be the exterior of this knot in $M$. The homological sequence of the pair $(M, E)$ and the inclusion $u \in$ Tors $H$ imply that $b_{1}(E) \geq b_{1}(M)+1 \geq 2$. Therefore $\tau(E) \in \mathbb{Z}\left[H_{1}(E)\right]$. Pick a representative $a \in \mathbb{Z}\left[H_{1}(E)\right]$ of $\tau(E)$. Denote by $\iota$ the inclusion homomorphism $H_{1}(E) \rightarrow H_{1}(M)=H$ and denote by $\iota_{*}$ the induced ring homomorphism $\mathbb{Z}\left[H_{1}(E)\right] \rightarrow \mathbb{Z}[H]$. By [11, Theorem VII.1.4], we have $\iota_{*}(a)=(u-1) b$ where $b$ is a representative of $\tau(M)$. Note that the right hand side of (8) does not depend on the choice of $\tau$ in $\tau(M)$. Therefore without loss of generality we can assume that $\tau=b$.

Deforming, if necessary, $X$ in $M$, we can assume that $S=X \cap E$ is the complement in $X$ of a regular neighborhood of $\operatorname{sing}(X)$. Then $S$ is a proper surface in $E$ and $\chi_{-}(X)=\chi_{-}(S)$. The orientation of $\operatorname{Int}(X)$ induces an orientation of $S$. The oriented surface $S$ represents a relative homology class $s \in H_{2}(E, \partial E)$. By (7),

$$
\chi_{-}(X)=\chi_{-}(S) \geq \max _{h, h^{\prime} \in B_{a}}\left|h \cdot s-h^{\prime} \cdot s\right|,
$$

where $B_{a} \subset H_{1}(E)$ is the set of $a$-basic elements. Let $r \in \mathbb{Q}$ be the minimal element of the set $\{h \cdot s\}_{h \in B_{a}}$. Then

$$
\{h \cdot s\}_{h \in B_{a}} \subset\left[r, r+\chi_{-}(X)\right] .
$$

Denote the projection $\mathbb{Q} \rightarrow \mathbb{Q} / \mathbb{Z}$ by $\pi$. Observe that for any $h \in H_{1}(E)$,

$$
\iota(h) \cdot x=\pi\left(\frac{h \cdot s}{i_{X}}\right) .
$$

Therefore $\quad\{\iota(h) \cdot x\}_{h \in B_{a}} \subset \pi\left(\left[\frac{r}{i_{X}}, \frac{r+\chi_{-}(X)}{i_{X}}\right]\right)$.

The equality $\iota_{*}(a)=(u-1) \tau$ implies that $B_{(u-1) \tau} \subset \iota\left(B_{a}\right)$. Hence

$$
\{g \cdot x\}_{g \in B_{(u-1) \tau}} \subset\{\iota(h) \cdot x\}_{h \in B_{a}} \subset \pi\left(\left[\frac{r}{i_{X}}, \frac{r+\chi_{-}(X)}{i_{X}}\right]\right) .
$$

Therefore

$$
\operatorname{spn}_{x}((u-1) \tau) \leq\left(i_{X}\right)^{-1} \chi_{-}(X) .
$$

Since this holds for all simple folded surfaces $X$ representing $x$, we have (8). 


\subsection{An estimate for $\Theta_{M}$}

Let $M$ and $H$ be as in Theorem 4.1. To estimate the function $\Theta_{M}$ : Tors $H \rightarrow \mathbb{Q} / \mathbb{Z}$, we need the linking form $L_{M}$ : Tors $H \times$ Tors $H \rightarrow \mathbb{Q} / \mathbb{Z}$ of $M$. It is defined by $L_{M}(h, g)=h \cdot x \in \mathbb{Q} / \mathbb{Z}$ where $x$ is an arbitrary element of $H_{2}(M ; \mathbb{Q} / \mathbb{Z})$ mapped to $g$ by the boundary homomorphism $d: H_{2}(M ; \mathbb{Q} / \mathbb{Z}) \rightarrow H$. The pairing $L_{M}$ is well defined, bilinear, and symmetric.

Given $u \in$ Tors $H$ and $a \in \mathbb{Q}[H]$, set

$$
\operatorname{spn}_{u}(a)=\operatorname{spn}\left(\left\{L_{M}(h, u)\right\}_{h \in B_{a} \cap \operatorname{Tors} H}\right) .
$$

Clearly, $\operatorname{spn}_{x}(a) \geq \operatorname{spn}_{d(x)}(a)$ for any $x \in H_{2}(M ; \mathbb{Q} / \mathbb{Z})$ and any $a \in \mathbb{Q}[H]$. This and Theorem 4.1 imply that, under the conditions of this theorem,

$$
\Theta_{M}(u) \geq \operatorname{spn}_{u}((u-1) \tau),
$$

for any $u \in$ Tors $H$ and any representative $\tau$ of $\tau(M)$. Generally speaking, the right-hand side of (9) depends on the choice of $\tau$.

Remark Estimate (7) strengthens the McMullen estimate [6] of the Thurston norm via the Alexander polynomial. For recent more general estimates of this type, see Friedl [3].

\section{Estimates from below: the case of $\mathbb{Q}$-homology spheres}

For $\mathbb{Q}$-homology spheres, the functions $\theta$ and $\Theta$ contain the same information and it is enough to give an estimate for $\Theta$. We begin with preliminaries on refined torsions and $\mathbb{Q}$-homology spheres, referring for details to [11, Chapters I and X].

\subsection{Refined torsions}

The maximal abelian torsion $\tau(M)$ of a compact connected 3-manifold $M$ admits a refinement $\tau(M, e, \omega) \in Q\left(H_{1}(M)\right)$ depending on an orientation $\omega$ in the vector space $H_{*}(M ; \mathbb{Q})=\oplus_{i \geq 0} H_{i}(M ; \mathbb{Q})$ and an Euler structure $e$ on $M$. An Euler structure on $M$ is determined by a nonsingular vector field on $M$ directed outside on $\partial M$. Two such vector fields determine the same Euler structure if for a point $x \in \operatorname{Int}(M)$, the restrictions of these fields to $M-\{x\}$ are homotopic in the class of nonsingular vector field on $M-\{x\}$ directed outside on $\partial M$. The set of Euler structures on $M$ is denoted $\operatorname{Eul}(M)$. This set admits a canonical free transitive action of the group $H_{1}(M)$. The torsion $\tau(M, e, \omega)$ satisfies $\tau(M, h e, \pm \omega)= \pm h \tau(M, e, \omega)$ for any $e \in \operatorname{Eul}(M), h \in H_{1}(M)$. The unrefined torsion $\tau(M)$ is just the set $\{ \pm \tau(M, e, \omega)\}_{e \in \operatorname{Eul}(M)}$. If $\partial M=\varnothing$, then the set $\operatorname{Eul}(M)$ can be identified with the set of $\operatorname{Spin}^{c}$-structures on $M$. 


\subsection{Homology spheres}

Let $M$ be an oriented 3-dimensional $\mathbb{Q}$-homology sphere. Denote $\omega_{M}$ the orientation in $H_{*}(M ; \mathbb{Q})=H_{0}(M ; \mathbb{Q}) \oplus H_{3}(M ; \mathbb{Q})$ determined by the following basis: (the homology class of a point, the fundamental class of $M)$.

The group $H=H_{1}(M)$ is finite and the linking form $L_{M}: H \times H \rightarrow \mathbb{Q} / \mathbb{Z}$ is nondegenerate in the sense that the adjoint homomorphism $H \rightarrow \operatorname{Hom}(H, \mathbb{Q} / \mathbb{Z})$ is an isomorphism. Recall that we use multiplicative notation for the group operation in $H$. Every Euler structure $e \in \operatorname{Eul}(M)$ determines a torsion $\tau\left(M, e, \omega_{M}\right) \in Q(H)=\mathbb{Q}[H]$. The linking form $L_{M}$ can be computed from this torsion by

$$
L_{M}(h, g)=-\pi\left(\left((1-h)(1-g) \tau\left(M, e, \omega_{M}\right)\right)_{1}\right) \in \mathbb{Q} / \mathbb{Z}
$$

for all $h, g \in H$, where $\pi$ is the projection $\mathbb{Q} \rightarrow \mathbb{Q} / \mathbb{Z}$ and for any $a \in \mathbb{Q}[H]$, the symbol $a_{1} \in \mathbb{Q}$ denotes the coefficient of the neutral element $1 \in H$ in the expansion of $a$ as a formal linear combination of elements of $H$ with rational coefficients. The Euler structure $e$ determines a function $q_{e}: H \rightarrow \mathbb{Q} / \mathbb{Z}$ by

$$
q_{e}(u)=\pi\left(\left((1-u) \tau\left(M, e, \omega_{M}\right)\right)_{1}\right),
$$

for any $u \in H$. It follows from (10) and (11) that $q_{e}$ is quadratic in the sense that $q_{e}(h g)=q_{e}(h)+q_{e}(g)+L_{M}(h, g)$ for all $h, g \in H$. Formula (11) also implies that

$$
q_{h e}(u)=q_{e}(u)+L_{M}(h, u),
$$

for any $h \in H$.

If $u \in H$ has order $n$ (ie $n$ is the minimal positive integer such that $u^{n}=1$ ), then by $[11$, Section X.4.3] there is a unique residue $K(e, u) \in \mathbb{Z} / 2 n \mathbb{Z}$ such that

$$
q_{e}(u)=\frac{K(e, u)}{2 n}+\frac{1}{2}(\bmod \mathbb{Z}) .
$$

Formula (12) implies that the residue $K(e, u)(\bmod 2)$ does not depend on $e$. We say that $u$ is even if this residue is 0 and odd if it is 1 .

Every homology class $u \in H$ gives rise to a group

$$
G=G_{u}=\left\{g \in H \mid L_{M}(u, g)=0\right\} \subset H .
$$

The nondegeneracy of $L_{M}$ implies that the quotient $H / G$ is a finite cyclic group whose order is equal to the order, $n$, of $u$ in $H$. Moreover, there is an element $v=v_{u} \in H$ such that $L_{M}(u, v)=n^{-1}(\bmod \mathbb{Z})$. Such $v$ is determined by $u$ uniquely 
up to multiplication by elements of $G$. The inclusion $v^{n} \in G$ implies that the order, $p$, of $v$ is divisible by $n$ (in particular, $p \geq n$ ). Set

$$
\alpha_{v}=\frac{1+2 v+3 v^{2}+\ldots+p v^{p-1}}{p}-\frac{p+1}{2} \cdot \frac{1+v+\ldots+v^{p-1}}{p} .
$$

This element of $\mathbb{Q}[H]$ can be uniquely characterized by the following property: if $\varphi$ is any ring homomorphism from $\mathbb{Q}[H]$ to a field, then $\varphi(v)=1 \Rightarrow \varphi\left(\alpha_{v}\right)=0$ and $\varphi(v) \neq 1 \Rightarrow \varphi\left(\alpha_{v}\right)=(\varphi(v)-1)^{-1}$. In [11] we used the notation $(v-1)_{\mathrm{par}}^{-1}$ for $\alpha_{v}$.

Theorem 5.1 Let $M$ be an oriented 3-dimensional $\mathbb{Q}$-homology sphere. Let $u$ be an element of $H=H_{1}(M)$ of order $n \geq 1$. Set $G=\left\{g \in H \mid L_{M}(u, g)=0\right\}$ and $\Sigma_{G}=\sum_{g \in G} g \in \mathbb{Z}[G] \subset \mathbb{Z}[H]$. Pick any $v \in H$ such that $L_{M}(u, v)=n^{-1}(\bmod 1)$. For $e \in \operatorname{Eul}(M)$, set

$$
a_{e}(u)=(u-1) \tau\left(M, e, \omega_{M}\right)-\frac{v^{K(e, u) / 2}(v+1)}{2} \alpha_{v} \Sigma_{G} \in \mathbb{Q}[H],
$$

if $u$ is even and

$$
a_{e}(u)=(u-1) \tau\left(M, e, \omega_{M}\right)-v^{(K(e, u)+1) / 2} \alpha_{v} \Sigma_{G} \in \mathbb{Q}[H],
$$

if $u$ is odd. Then for any $e \in \operatorname{Eul}(M)$,

$$
\Theta_{M}(u) \geq \operatorname{spn}_{u}\left(a_{e}(u)\right)=\operatorname{spn}\left(\left\{L_{M}(h, u)\right\}_{h \in B_{a_{e}(u)}}\right) .
$$

Proof If $u$ is even (resp. odd), then $K(e, u) \in \mathbb{Z}_{2 n}$ is even (resp. odd). Therefore the power of $v$ in the definition of $a_{e}(u)$ is well defined up to multiplication by $v^{n}$. However, $v^{n} \in G$ and $v^{n} \Sigma_{G}=\Sigma_{G}$. Therefore the right hand sides of the formulas for $a_{e}(u)$ are well defined. If $v^{\prime}$ is another element of $H$ such that $L_{M}\left(u, v^{\prime}\right)=$ $n^{-1}(\bmod 1)$, then $v^{\prime} \in v G$ and $v^{k} \Sigma_{G}=\left(v^{\prime}\right)^{k} \Sigma_{G}$ for all $k \in \mathbb{Z}$. Therefore $a_{e}(u)$ does not depend on the choice of $v$. It is easy to see that $a_{h e}(u)=h a_{e}(u)$ for all $h \in H$. Therefore the number $\operatorname{spn}_{u}\left(a_{e}(u)\right)$ does not depend on $e$.

Consider a simple folded surface $X \subset M$ which represents the 2-homology class $x=d_{M}^{-1}(u) \in H_{2}(M ; \mathbb{Q} / \mathbb{Z})$. The knot $K=\operatorname{sing}(X)$ with orientation induced from the one on $X$ represents $u \in H_{1}(M)$. Let $E$ be the exterior of $K$ in $M$. Clearly $b_{1}(E)=1$. Fix an orientation $\omega$ in $H_{*}(E ; \mathbb{Q})$ and an Euler structure $e_{K}$ on $E$. The torsion $\tau\left(E, e_{K}, \omega\right) \in Q\left(H_{1}(E)\right)$ can be canonically expanded as a sum of a certain $[\tau]=[\tau]\left(E, e_{K}, \omega\right) \in \mathbb{Q}\left[H_{1}(E)\right]$ with an element of $Q\left(H_{1}(E)\right)$ given by an explicit formula using solely $\omega$ and the Chern class of $e_{K}$ [11, Section II.4.5]. The inclusion homomorphism $\mathbb{Q}\left[H_{1}(E)\right] \rightarrow \mathbb{Q}\left[H_{1}(M)\right]$ sends $[\tau]$ to $\pm a_{e}(u)$ for some $e \in \operatorname{Eul}(M)$ [11, Formula X.4.d]. The inequality (7) holds for any $s \in H_{2}(E, \partial E ; \mathbb{Q})$ and $a=[\tau]$ 
[11, Chapter IV]. The rest of the argument goes as the proof of Theorem 4.1 with $\tau$ replaced by $[\tau]$. This gives $\left(i_{X}\right)^{-1} \chi_{-}(X) \geq \operatorname{spn}_{x}\left(a_{e}(u)\right)=\operatorname{spn}_{u}\left(a_{e}(u)\right)$. Since this holds for all $X$ representing $x$, we have $\Theta_{M}(u)=\theta_{M}(x) \geq \operatorname{spn}_{u}\left(a_{e}(u)\right)$.

Remarks 1. Let $\frac{1}{2} \mathbb{Z}$ be the additive group of integers and half-integers. Then in Theorem 5.1, $a_{e}(u) \in \mathbb{Z}[H]$ if $u$ is even and $a_{e}(u) \in \frac{1}{2} \mathbb{Z}[H]$ if $u$ is odd. This follows from the proof of this theorem and the inclusion $[\tau] \in \mathbb{Z}\left[H_{1}(E)\right]$ if $u$ is even and $[\tau] \in \frac{1}{2} \mathbb{Z}\left[H_{1}(E)\right]$ if $u$ is odd.

2. It is proven by Deloup and Massuyeau [2] that the function $q_{e}: H \rightarrow \mathbb{Q} / \mathbb{Z}$ derived from the torsion coincides with the quadratic function defined geometrically by Looijenga and Wahl [5] and Deloup [1].

\section{Examples}

\subsection{Lens spaces}

The computation of the abelian torsions for the lens space $M=L(p, q)$ goes back to K Reidemeister. See, for instance, [10] for an introduction to the theory of torsions. Let $t, t^{q}$ be the generators of $H=H_{1}(M)$ represented by the core circles of the two solid tori forming $M$. For an appropriate choice of an orientation on $M$ and an Euler structure $e$ on $M$, we have $\tau\left(M, e, \omega_{M}\right)=\alpha_{t} \alpha_{t} q$, where $\alpha_{v} \in \mathbb{Q}[H]$ is defined by (14) for any $v \in H$. This allows us to compute $a_{e}(u)$ for any $u \in H$ and to apply Theorem 5.1. We give here a few examples.

Consider the lens space $M=L(5,1)$. By Section 2.3 and Section 2.4.2, $\Theta$ satisfies $\Theta\left(t^{4}\right)=\Theta(t)=\Theta(1)=0$ and $\Theta\left(t^{2}\right)=\Theta\left(t^{3}\right)$. We show that $\Theta\left(t^{2}\right) \geq 1 / 5$. We have

$$
\alpha_{t}=\frac{-2-t+t^{3}+2 t^{4}}{5} \text {. }
$$

Then

$$
\tau=\tau\left(M, e, \omega_{M}\right)=\alpha_{t}^{2}=\frac{t+t^{2}-2 t^{4}}{5} .
$$

A direct computation shows that

$$
L_{M}(t, t)=\left(-(1-t)^{2} \tau\right)_{1}=1 / 5, \quad q_{e}\left(t^{2}\right)=((1-t) \tau)_{1}=0 .
$$

Note that $u=t^{2}$ has order 5 in $H$. From (13), we obtain that $K(e, u)=5(\bmod 10)$. Therefore $u$ is odd. The associated group $G_{u}$ is trivial, $v=v_{u}=t^{3}$, and

$$
a_{e}(u)=(u-1) \tau-v^{3} \alpha_{v}=t^{4}-t .
$$


Since $L_{M}\left(t^{4}, u\right)=3 / 5(\bmod 1)$ and $L_{M}(t, u)=2 / 5(\bmod 1)$, the span of the set $\left\{L_{M}(h, u)\right\}_{h \in B_{a_{e}(u)}}$ is equal to $1 / 5$. By Theorem 5.1, $\Theta\left(t^{2}\right) \geq 1 / 5$.

Consider the lens space $M=L(6,1)$. Then

$$
\begin{gathered}
\alpha_{t}=\frac{-5-3 t-t^{2}+t^{3}+3 t^{4}+5 t^{5}}{12}, \\
\tau=\alpha_{t}^{2}=\frac{-5+13 t+19 t^{2}+13 t^{3}-5 t^{4}-35 t^{5}}{72},
\end{gathered}
$$

and $L_{M}(t, t)=1 / 6$. For $u=t^{2}$, the computations similar to the ones above give $q_{e}(u)=0(\bmod 1), K(e, u)=3(\bmod 6), G_{u}=\left\{1, t^{3}\right\}, v_{u}=t$, and $a_{e}(u)=t^{5}-t$. Theorem 5.1 yields $\Theta\left(t^{2}\right) \geq 1 / 3$. For $u=t^{3}$, we similarly obtain $q_{e}(u)=3 / 4(\bmod 1)$, $K(e, u)=1(\bmod 4), G_{u}=\left\{1, t^{2}, t^{4}\right\}, v_{u}=t$, and $a_{e}(u)=t^{5}-t^{2}$. Theorem 5.1 yields $\Theta\left(t^{3}\right) \geq 1 / 2$.

\subsection{Surgeries on knots}

Let $L$ be an oriented knot in an oriented 3-dimensional $\mathbb{Z}$-homology sphere $N$. Let $M$ be the closed oriented 3-manifold obtained by surgery on $N$ along $L$ with framing $p \geq 2$. Let $u \in H=H_{1}(M)$ be the homology class of the meridian of $L$ whose linking number with $L$ is +1 . Clearly, $H$ is a cyclic group of order $p$ with generator $u$ and $L_{M}(u, u)=p^{-1}(\bmod 1)$. We explain now how to estimate $\Theta(u)$ in terms of the Alexander polynomial of $L$. We will see that in some cases this estimate is exact.

Recall that the span $\operatorname{spn}(\Delta)$ of a nonzero Laurent polynomial $\Delta=\sum_{i} a_{i} t^{i} \in \mathbb{Z}\left[t^{ \pm 1}\right]$ is the number $\max \left\{i \mid a_{i} \neq 0\right\}-\min \left(\left\{i \mid a_{i} \neq 0\right\}\right)$. Let $\Delta=\Delta_{L}(t)$ be the Alexander polynomial of $L$ normalized so that $\Delta\left(t^{-1}\right)=\Delta(t)$ and $\Delta(1)=1$. Expand $\Delta(t)=1+(t-1) \beta(t)$ where $\beta(t) \in \mathbb{Z}\left[t^{ \pm 1}\right]$. We claim the expression $a_{e}(u) \in \mathbb{Q}[H]$ defined in Theorem 5.1 is equal to $\beta(u)$ for an appropriate Euler structure $e$ on $M$. By Theorem 5.1, this will imply that $\Theta(u) \geq \operatorname{spn}_{u}(\beta(u))$. For example, if $p \geq 2 \operatorname{spn}(\beta)$, then $\operatorname{spn}_{u}(\beta(u))=p^{-1} \operatorname{spn}(\beta)=p^{-1}(\operatorname{spn}(\Delta)-1)$. Therefore $\Theta(u) \geq p^{-1}(\operatorname{spn}(\Delta)-1)$. On the other hand, by Section 2.4.3, $\Theta(u) \leq p^{-1}(2 g-1)$, where $g$ is the genus of $K$. In particular, if $\operatorname{spn}(\Delta)=2 g>0$ (for instance, if $K$ is a nontrivial fibred knot) and $p \geq 4 g-2$, then $\Theta(u)=p^{-1}(2 g-1)$.

We now verify the claim above. Set $\tau=\alpha_{u}^{2} \Delta(u) \in \mathbb{Q}[H]$. It is easy to deduce from the multiplicativity of the torsions that $\tau\left(M, e, \omega_{M}\right)=\tau$ for a certain orientation on $M$ and a certain Euler structure $e$ on $M$ (for details, see [11, Formula X.5.e]). Set $\sigma=1+u+u^{2}+\cdots+u^{p-1} \in \mathbb{Z}[H]$. Clearly, $\sigma u^{k}=\sigma$ for any integer $k$. Therefore for any integer 1 -variable polynomial $f$, the product $\sigma f(u)$ is equal to $\operatorname{aug}(f) \sigma$ 
where $\operatorname{aug}(f)=f(1)$ is the sum of coefficients of $f$. Since $\operatorname{aug}\left(\alpha_{u}\right)=0$, we have $\sigma \alpha_{u}=0$. A direct computation shows that $(1-u) \alpha_{u}=\sigma / p-1$. Hence

$$
\begin{aligned}
(1-u) \tau & =(1-u) \alpha_{u}^{2} \Delta(u)=(\sigma / p-1) \alpha_{u} \Delta(u)=-\alpha_{u} \Delta(u) \\
& =-\alpha_{u}+\alpha_{u}(1-u) \beta(u)=-\alpha_{u}+(\sigma / p-1) \beta(u)=-\alpha_{u}-\beta(u),
\end{aligned}
$$

where we use the equality $\operatorname{aug}(\beta)=0$ which follows from the symmetry of $\Delta$. Thus,

$$
q_{e}(u)=((1-u) \tau)_{1}=-\left(\alpha_{u}\right)_{1}=(p-1) / 2 p(\bmod 1) .
$$

Formula (13) implies that $K(e, u)=-1(\bmod 2 p)$. In particular, $u$ is odd.

We also have

$$
(1-u)^{2} \tau=(1-u)\left(-\alpha_{u}-\beta(u)\right)=1-\sigma / p-(1-u) \beta(u) .
$$

Hence

$$
L_{M}(u, u)=-\left((1-u)^{2} \tau\right)_{1}=p^{-1}(\bmod 1) .
$$

This shows that the orientation of $M$ chosen so that $\tau\left(M, e, \omega_{M}\right)=\tau$ is actually the one induced from the orientation on $N$. The equality $L_{M}(u, u)=p^{-1}(\bmod 1)$ implies that $v_{u}=u$ and $G_{u}=1$. We conclude that

$$
a_{e}(u)=(u-1) \tau-\alpha_{u}=\alpha_{u}+\beta(u)-\alpha_{u}=\beta(u) .
$$

\subsection{Surgeries on 2-component links}

Let $M$ be a closed oriented 3-manifold obtained by surgery on a 2-component oriented link $L=L_{1} \cup L_{2}$ in an oriented 3-dimensional $\mathbb{Z}$-homology sphere $N$. Suppose that the linking number of $L_{1}, L_{2}$ in $N$ is 0 , the framing of $L_{1}$ is $p \neq 0$, and the framing of $L_{2}$ is 0 . Then $H=H_{1}(M)=(\mathbb{Z} / p \mathbb{Z}) u_{1} \oplus \mathbb{Z} u_{2}$, where $u_{i} \in H$ is the homology class of the meridian of $L_{i}$ whose linking number with $L_{i}$ is +1 , for $i=1,2$. The Alexander polynomial of $L$ has the form

$$
\Delta_{L}\left(t_{1}, t_{2}\right)=f\left(t_{1}, t_{2}\right)\left(t_{1}-1\right)\left(t_{2}-1\right)
$$

for some Laurent polynomial $f\left(t_{1}, t_{2}\right) \in \mathbb{Z}\left[t_{1}^{ \pm 1}, t_{2}^{ \pm 1}\right]$. Both $\Delta_{L}$ and $f$ are defined only up to multiplication by -1 and monomials on $t_{1}, t_{2}$. By [11, Formula VIII.4.e], the torsion $\tau(M)$ is represented by

$$
\tau=f\left(u_{1}, u_{2}\right) \pm \Delta_{L_{2}}\left(u_{2}\right) u_{2}^{n}\left(u_{2}-1\right)^{-2} \Sigma_{H} \in Q(H)
$$

for an appropriate sign \pm and an integer $n$, both depending on the choice of $f$. Here $\Delta_{L_{2}}$ is the Alexander polynomial of $L_{2}$ normalized as in Section 6.2. Pick 
$x \in H_{2}(M ; \mathbb{Q} / \mathbb{Z})$ and set $u=d(x) \in$ Tors $H$. Since $(u-1) \Sigma_{H}=0$, Theorem 4.1 implies that

$$
\theta(x) \geq \operatorname{spn}_{x}\left((u-1) f\left(u_{1}, u_{2}\right)\right) .
$$

For sufficiently big $p$, the span on the right hand side does not depend on $p$.

Note another curious phenomenon. Suppose for simplicity that $f\left(t_{1}, t_{2}\right)=1$ (a constant polynomial). Then $\theta(x) \geq \operatorname{spn}_{x}(u-1)$. If $u=d(x) \neq 1$, then the set $B_{u-1} \subset H$ consists of two elements $u, 1$ and

$$
\operatorname{spn}_{x}(u-1)=\operatorname{spn}(\{u \cdot x, 0\})=\operatorname{spn}\left(\left\{L_{M}(u, u), 0\right\}\right) .
$$

For $u=u_{1}^{k}$ with $k \in\{0,1, \ldots, n-1\}$, we have $L_{M}(u, u)=k^{2} / n(\bmod 1)$. For $k<\sqrt{n / 2}$, we obtain $\operatorname{spn}\left(\left\{L_{M}(u, u), 0\right\}\right)=k^{2} / n$. Thus $\Theta\left(u_{1}^{k}\right) \geq k^{2} / n$. This suggests that the number $\Theta\left(u_{1}^{k}\right)$, considered as a function of $k$, may behave like a quadratic function for small values of $k$.

\section{Miscellaneous}

\subsection{Quasi-simple folded surfaces}

One can use a larger class of folded surfaces to represent 2-homology classes. Let us call a folded surface $X$ quasi-simple if it is oriented, $\operatorname{sing}(X) \neq \varnothing$, and the indices of all components of $\operatorname{sing}(X)$ in $X$ are equal to each other and nonzero. Denote the common value of these indices $i_{X}$. In particular, simple folded surfaces are quasi-simple.

For a quasi-simple folded surface $X$ in a 3-manifold $M$, the 2 -chain $\left(i_{X}\right)^{-1} X$ is a 2 -cycle $\bmod \mathbb{Z}$ representing a homology class $[X] \in H_{2}(M ; \mathbb{Q} / \mathbb{Z})$. We claim that

$$
\theta([X]) \leq i_{X}^{-1} \chi_{-}(X)+b_{0}(\operatorname{sing}(X))-1,
$$

where $b_{0}(\operatorname{sing}(X))$ is the number of components of $\operatorname{sing}(X)$. Indeed, $X$ can be modified in a neighborhood of $\operatorname{sing}(X)$ so that each point of $\operatorname{sing}(X)$ is adjacent to exactly $i_{X}$ local branches of $\operatorname{Int}(X)$ (which then induce the same orientation on $\operatorname{sing}(X))$. Let $\Gamma$ be a graph with two vertices and $i_{X}$ edges connecting these vertices. Given an embedded arc in $M$ with endpoints on different components of $\operatorname{sing}(X)$ and with interior in $M-X$, we can modify $X$ by cutting it out along $\operatorname{sing}(X)$ near the endpoints and gluing in $\Gamma \times[0,1]$ along the arc. This gives a quasi-simple folded surface, $Z$, such that

$b_{0}(\operatorname{sing}(Z))=b_{0}(\operatorname{sing}(X))-1, \quad i_{Z}=i_{X}, \quad[Z]=[X], \quad$ and $\quad \chi_{-}(Z) \leq \chi_{-}(X)+i_{X}$. 
Modifying $X$ in this way, we can reduce ourselves to the case where $\operatorname{sing}(X)$ is connected. In this case (16) follows from the definition of $\theta$. It may happen that there are no distinct components of $\operatorname{sing}(X)$ connected by an arc with interior in $M-X$. This occurs if each arc joining distinct components of $\operatorname{sing}(X)$ has to cross the closed 2-manifold $X_{0}$ formed by the components of $X$ disjoint from $\operatorname{sing}(X)$. To circumvent this obstruction, we first modify $X_{0}$ so that $X-X_{0}$ is contained in a connected component of $M-X_{0}$; cf [11, p 60].

Formula (16) implies that for any $x \in H_{2}(M ; \mathbb{Q} / \mathbb{Z})$,

$$
\theta(x)=\inf _{X}\left(\frac{\chi_{-}(X)}{i_{X}}+b_{0}(\operatorname{sing}(X))\right)-1,
$$

where $X$ runs over all quasi-simple folded surfaces in $M$ representing $x$.

\subsection{Coverings}

Let $M$ be a compact oriented 3-manifold and $p: \widetilde{M} \rightarrow M$ be an $n$-fold (unramified) covering. Let $p^{*}: H_{2}(M ; \mathbb{Q} / \mathbb{Z}) \rightarrow H_{2}(\widetilde{M} ; \mathbb{Q} / \mathbb{Z})$ be the following composition of the duality isomorphisms and the pull back

$$
H_{2}(M ; \mathbb{Q} / \mathbb{Z}) \cong H^{1}(M, \partial M ; \mathbb{Q} / \mathbb{Z}) \rightarrow H^{1}(\widetilde{M}, \partial \widetilde{M} ; \mathbb{Q} / \mathbb{Z}) \cong H_{2}(\widetilde{M} ; \mathbb{Q} / \mathbb{Z}) .
$$

Then for any $x \in H_{2}(M ; \mathbb{Q} / \mathbb{Z})$,

$$
\theta_{\widetilde{M}}\left(p^{*}(x)\right)+1 \leq n\left(\theta_{M}(x)+1\right) .
$$

This follows from (17) and the fact that if a simple folded surface $X$ in $M$ represents $x$, then $p^{-1}(X) \subset \widetilde{M}$ is a quasi-simple folded surface representing $p^{*}(x)$.

\subsection{Norms associated with links}

A link $L$ in an oriented 3-manifold $M$ determines a semi-norm $\|\cdot\|_{M, L}$ on $H_{2}(M ; \mathbb{Q})$ as follows. Let $U \subset M$ be a regular neighborhood of $L$ and $E=\overline{M-U}$ the exterior of $L$. We can embed $H_{2}(M ; \mathbb{Q})$ into $H_{2}(E, \partial E ; \mathbb{Q})$ via the inclusion homomorphism

$$
H_{2}(M ; \mathbb{Q}) \hookrightarrow H_{2}(M, L ; \mathbb{Q}) \cong H_{2}(M, U ; \mathbb{Q}) \cong H_{2}(E, \partial E ; \mathbb{Q}) .
$$

Restricting the Thurston semi-norm on $H_{2}(E, \partial E ; \mathbb{Q})$ to $H_{2}(M ; \mathbb{Q})$, we obtain the semi-norm $\|\cdot\|_{M, L}$. The arguments as above allow us to estimate the latter semi-norm from below for compact $M$. Namely, if $L$ has $m \geq 1$ components and $h_{1}, \ldots, h_{m}$ are their homology classes in $H=H_{1}(M)$, then

$$
\|x\|_{M, L} \geq \operatorname{spn}_{x}\left(\prod_{i=1}^{m}\left(h_{i}-1\right) \tau\right)
$$

Algebraic 83 Geometric Topology, Volume 7 (2007) 
for any $x \in H_{2}(M ; \mathbb{Q})$ and any $\tau \in Q(H)$ representing $\tau(M)$ in the case $b_{1}(M) \geq 2$ and representing $[\tau](M)$ in the case $b_{1}(M)=1$. A similar construction can be used to derive a function on $H_{2}(M ; \mathbb{Q} / \mathbb{Z})$ from the function $\theta$ on $H_{2}(E, \partial E ; \mathbb{Q} / \mathbb{Z})$. It would be interesting to see whether these semi-norms and functions may be used to distinguish nonisotopic links.

\subsection{Open questions}

Is the infimum in (2) realizable by a simple folded surface? Does $\theta$ take only rational values? A positive answer to the first question certainly implies a positive answer to the second one. Similar questions can be asked for $\Theta$.

It would be interesting to compute the function $\Theta$ for the lens spaces. Is it true that for the lens spaces, the inequality in Theorem 5.1 is an equality?

\section{References}

[1] F Deloup, On abelian quantum invariants of links in 3-manifolds, Math. Ann. 319 (2001) 759-795 MR1825407

[2] F Deloup, G Massuyeau, Reidemeister-Turaev torsion modulo one of rational homology three-spheres, Geom. Topol. 7 (2003) 773-787 MR2026547

[3] S Friedl, Reidemeister torsion, the Thurston norm and Harvey's invariants arXiv: math.GT/0508648

[4] P B Kronheimer, TS Mrowka, Scalar curvature and the Thurston norm, Math. Res. Lett. 4 (1997) 931-937 MR1492131

[5] E Looijenga, J Wahl, Quadratic functions and smoothing surface singularities, Topology 25 (1986) 261-291 MR842425

[6] C T McMullen, The Alexander polynomial of a 3-manifold and the Thurston norm on cohomology, Ann. Sci. École Norm. Sup. (4) 35 (2002) 153-171 MR1914929

[7] L I Nicolaescu, The Reidemeister torsion of 3-manifolds, de Gruyter Studies in Mathematics 30, Walter de Gruyter \& Co., Berlin (2003) MR1968575

[8] P Ozsváth, Z Szabó, Holomorphic disks and genus bounds, Geom. Topol. 8 (2004) 311-334 MR2023281

[9] W P Thurston, A norm for the homology of 3-manifolds, Mem. Amer. Math. Soc. 59 (1986) i-vi and 99-130 MR823443

[10] V Turaev, Introduction to combinatorial torsions, Lectures in Mathematics ETH Zürich, Birkhäuser Verlag, Basel (2001) MR1809561Notes taken by Felix Schlenk 
[11] V Turaev, Torsions of 3-dimensional manifolds, Progress in Mathematics 208, Birkhäuser Verlag, Basel (2002) MR1958479

IRMA, CNRS et Université Louis Pasteur

7 rue René Descartes, 67084 Strasbourg, France

Department of Mathematics, Indiana University

Bloomington IN 47405, USA

Received: 21 November 2006

Algebraic $8 \mathcal{G}$ Geometric Topology, Volume 7 (2007) 